\title{
An Example Concerning the Translative Kissing Number of a Convex Body*
}

\section{Chuanming Zong}

Institut für Analysis, Technische Mathematik und Versicherungsmathematik, Wiedner Hauptstrasse 8-10, A-1040 Wien, Austria

and

Institute of Mathematics, The Chinese Academy of Sciences,

Beijing 100080, People's Republic of China.

\begin{abstract}
This article presents a class of convex bodies in $E^{d}(d \geq 3)$ where their maximum kissing numbers in translative packings are larger than their maximum kissing numbers in lattice packings.
\end{abstract}

\section{Introduction}

Let $K$ be a convex body, let $N_{\mathrm{T}}(K)$ be the maximum kissing number of $K$ in translative packings, and let $N_{\mathrm{L}}(K)$ be the maximum kissing number of $K$ in lattice packings. In other words, $N_{\mathrm{T}}(K)$ is the maximum number of the nonoverlapping translates of $K$ which can be brought into contact with $K$ and $N_{\mathrm{L}}(K)$ is the maximum number of the translates of $K$ in lattice packings which can be brought into contact with $K$.

Concerning $N_{\mathrm{T}}(K)$ and $N_{\mathrm{L}}(K)$, the following question can be asked:

Is there a convex body $K$ such that $N_{\mathrm{T}}(K)>N_{\mathrm{L}}(K)$ ?

In 1961 Grünbaum [3] proved a conjecture of Hadwiger which implies

$$
N_{\mathrm{T}}(K)=N_{\mathrm{L}}(K)
$$

\footnotetext{
* This work was supported by the Austrian Academic Exchange Service.
} 
for all convex domains $K$ in $E^{2}$. Unexpectedly, 10 years later Watson [4] found that the maximum kissing number of a nine-dimensional sphere in lattice packings is 272 while a translative packing exists in which some nine-dimensional spheres are touched by 306 others. In a summary of the known results about this problem, Conway and Sloane [1] in 1988 wrote: "As regards the maximum kissing number, 9 is the first dimension in which nonlattice packings are known to be superior to lattice packings."

In this article we prove that when $d \geq 3$ there are always $d$-dimensional convex bodies $K$ such that

$$
N_{\mathrm{T}}(K)>N_{\mathrm{L}}(K)
$$

By this we solve our problem completely with regard to the dimensions.

\section{The Three-Dimensional Case}

In this section we present an example in $E^{3}$. Defining

$$
U=\left\{\left(x^{1}, x^{2}, x^{3}\right):\left|x^{i}\right| \leq \frac{1}{2}, i=1,2,3\right\}
$$

and

$$
V=\left\{\left(x^{1}, x^{2}, x^{3}\right):\left|x^{i}\right| \leq \frac{1}{2}, i=1,2,3,\left|x^{1}+x^{2} \pm x^{3}\right| \leq \frac{4}{3}\right\}
$$

we show that $V$ is a required example. Namely, we prove

Theorem 1. $N_{\mathrm{L}}(V)=22<24 \leq N_{\mathrm{T}}(V)$.

In order to carry out the proof of Theorem 1 efficiently, we first show a very special assertion which plays a key role in the proof of $N_{\mathrm{L}}(K)=22$.

Assertion Z. Denote by $W$ the square $\left\{\left(x^{1}, x^{2}\right):\left|x^{1}\right| \leq \frac{1}{2},\left|x^{2}\right| \leq \frac{1}{2}\right\}$. Let $T$ be a point set in $E^{2}$ such that $o \notin T, W+T$ is a translative packing, and $W \cap(W+t) \neq \varnothing$ holds for every point $t \in T$, then we have

$$
\operatorname{card}\{T\} \leq 8
$$

and the equality holds only if

$$
\{(1,1),(1,-1),(-1,1),(-1,-1)\} \subset T \text {. }
$$


Sketch of the Proof. Let $T=\left\{t_{1}, t_{2}, \ldots, t_{n}\right\}, t_{i}=\left(t_{i}^{1}, t_{i}^{2}\right), \eta\left(t_{i}\right)=\max \left\{\left|t_{i}^{1}\right|,\left|t_{i}^{2}\right|\right\}$, $t_{i}^{*}=t_{i} / \eta\left(t_{i}\right), i=1,2, \ldots, n$, and $T^{*}=\left\{t_{1}^{*}, t_{2}^{*}, \ldots, t_{n}^{*}\right\}$. By considering the related positions of $t_{i}$ and $t_{j}$ it can be verified that $W+T^{*} \cup\{0\}$ is a translative packing, $W \cap\left(W+t^{*}\right) \neq \varnothing$ holds for every point $t^{*} \in T^{*}$, and $t_{i}^{*} \neq t_{j}^{*}$ if $i \neq j$. Then, recalling Groemer's well-known result on the kissing numbers (see [2]), we get

$$
\operatorname{card}\{T\}=\operatorname{card}\left\{T^{*}\right\} \leq 8
$$

and the equality holds only if

$$
T^{*}=\{(1,1),(1,0),(1,-1),(0,-1),(-1,-1),(-1,0),(-1,1),(0,1)\}
$$

Then, from the relationship between $T$ and $T^{*}$, we can see that (1) implies

$$
\{(1,1),(1,-1),(-1,-1),(-1,1)\} \subset T \text {. }
$$

Thus, our assertion is proved.

Lemma 1. $N_{\mathrm{T}}(V) \geq 24$.

Proof. Let $T$ be the set of points $(0,0,0),( \pm 1,0,0),(0, \pm 1,0),(0,0, \pm 1)$, $( \pm 1,0, \pm 1),(0, \pm 1, \pm 1), \pm(-1,1,1), \pm(1,-1,1), \pm(-1,1,0),\left(1,1, \pm \frac{1}{2}\right)$, and $\left(-1,-1, \pm \frac{1}{2}\right)$. It is easy to check that $V+T$ is a translative packing and

$$
(V+t) \cap V \neq \varnothing
$$

holds for every point $t \in T$. This means the kissing number of $V$ in the packing $V+T$ is 24 . This proves the lemma.

Lemma 2. $N_{\mathrm{L}}(V)=22$.

Proof. Let $\Lambda$ be a lattice such that $V+\Lambda$ is a packing, and let $N$ be the kissing number of $V$ in the lattice packing $V+\Lambda$. Now we prove our lemma by dealing with the following cases:

Case 1: All $(1,0,0),(0,1,0)$, and $(0,0,1)$ belong to $\Lambda$. In this case, $\Lambda$ is uniquely determined by these three points and it can be easily calculated that

$$
N=22 \text {. }
$$


Case 2: One of these three points $(1,0,0),(0,1,0)$, and $(0,0,1)$ does not belong to $\Lambda$. Without loss of generality, if $(0,0,1) \notin \Lambda$, we define

$$
\begin{aligned}
& J_{1}=\left\{x=\left(x^{1}, x^{2}, x^{3}\right): x \in \Lambda, x^{3}>\frac{1}{3},(V+x) \cap V \neq \varnothing\right\}, \\
& J_{2}=\left\{x=\left(x^{1}, x^{2}, x^{3}\right): x \in \Lambda \backslash\{o\},\left|x^{3}\right| \leq \frac{1}{3},(V+x) \cap V \neq \varnothing\right\},
\end{aligned}
$$

and

$$
J_{3}=\left\{x=\left(x^{1}, x^{2}, x^{3}\right): x \in \Lambda, x^{3}<-\frac{1}{3},(V+x) \cap V \neq \varnothing\right\}
$$

Obviously,

$$
N=\operatorname{card}\left\{J_{1}\right\}+\operatorname{card}\left\{J_{2}\right\}+\operatorname{card}\left\{J_{3}\right\}
$$

Now, we estimate card $\left\{J_{1}\right\}, \operatorname{card}\left\{J_{2}\right\}$, and card $\left\{J_{3}\right\}$, respectively, as follows (when $(1,0,0) \notin \Lambda$ or $(0,1,0) \notin \Lambda$, the three sets $J_{1}, J_{2}$, and $J_{3}$ can be defined according to $x^{1}$ or $x^{2}$, respectively, and can be estimated in a similar way):

(i) $\operatorname{card}\left\{J_{1}\right\}$. Let $H$ be the hyperplane $x^{3}=\frac{2}{3}$ and

$$
H\left(J_{1}\right)=\left\{\left(x^{1}, x^{2}, \frac{2}{3}\right):\left(x^{1}, x^{2}, x^{3}\right) \in J_{1}\right\}
$$

Keeping the definitions of $V$ and $J_{1}$ and the assumption that $(0,0,1) \notin J_{1}$ in mind, we see that

$$
\left(0,0, \frac{2}{3}\right) \notin H\left(J_{1}\right), \quad \operatorname{card}\left\{H\left(J_{1}\right)\right\}=\operatorname{card}\left\{J_{1}\right\},
$$

$\bigcup_{x \in J_{1}}((V+x) \cap H)=W+H\left(J_{1}\right)$ is a translative packing of $W$ in the hyperplane $H$ (different from the definition in Assertion $Z$, here $W$ indicates the square $\left.\left\{\left(x^{1}, x^{2}, 0\right):\left|x^{1}\right| \leq \frac{1}{2},\left|x^{2}\right| \leq \frac{1}{2}\right\}\right)$, and $\left(W+\left(0,0, \frac{2}{3}\right)\right) \cap(W+y) \neq \varnothing$ holds for every point $y \in H\left(J_{1}\right)$. So, by Assertion $Z$ we get

$$
\operatorname{card}\left\{J_{1}\right\}=\operatorname{card}\left\{H\left(J_{1}\right)\right\} \leq 8
$$

and the equality holds only if four points

$$
\left(1,1, \alpha_{1}\right),\left(-1,1, \alpha_{2}\right),\left(1,-1, \alpha_{3}\right),\left(-1,-1, \alpha_{4}\right) \in J_{1}
$$

exist. 
(ii) $\operatorname{card}\left\{J_{2}\right\}$ and card $\left\{J_{3}\right\}$. Apparently, the previous assumption $(0,0,1) \notin \Lambda$ implies $(0,0,-1) \notin J_{3}$. Thus by applying a similar argument to sets $J_{2}$ and $J_{3}$ we get

$$
\begin{aligned}
& \operatorname{card}\left\{J_{2}\right\} \leq 8, \\
& \operatorname{card}\left\{J_{3}\right\} \leq 8
\end{aligned}
$$

and the equalities in (6) and (7) hold only if there are points

$$
\left(1,1, \beta_{1}\right),\left(-1,1, \beta_{2}\right),\left(1,-1, \beta_{3}\right),\left(-1,-1, \beta_{4}\right) \in J_{2}
$$

and

$$
\left(1,1, \gamma_{1}\right),\left(-1,1, \gamma_{2}\right),\left(1,-1, \gamma_{3}\right),\left(-1,-1, \gamma_{4}\right) \in J_{3} .
$$

However, (5), (8), and (9) cannot hold simultaneously since the three nonoverlapping translates $V+\left(1,1, \alpha_{1}\right), V+\left(1,1, \beta_{1}\right)$, and $V+\left(1,1, \gamma_{1}\right)$ cannot be brought into contact with $V$ at the same time. So, the equalities in (4), (6), and (7) cannot hold simultaneously. Thus, combining with (3) we get

$$
N=\operatorname{card}\left\{J_{1}\right\}+\operatorname{card}\left\{J_{2}\right\}+\operatorname{card}\left\{J_{3}\right\}<24 .
$$

Considering the fact that the kissing number of $V$ in any lattice packing is an even number, (10) can be improved to

$$
N \leq 22
$$

Thus, by these two cases we get

$$
N_{\mathbf{L}}(V)=22
$$

Lemma 2 is proved.

Obviously, Theorem 1 is an immediate consequence of Lemmas 1 and 2.

\section{Two Generalizations and One Problem}

By a similar argument we can prove

Theorem 2. Let $K$ be a convex body such that $V \subset K \subset U$ and $\pm\left(\frac{1}{2}, \frac{1}{2}, \pm \frac{1}{2}\right) \notin K$, then

$$
N_{\mathrm{L}}(K)=22<24 \leq N_{\mathrm{T}}(K)
$$


In high dimensions we have the following analogy:

Theorem 3. If $d \geq 3$,

$$
\begin{aligned}
& K_{1}=\left\{x=\left(x^{1}, x^{2}, \ldots, x^{d}\right):\left|x^{i}\right| \leq \frac{1}{2},\left| \pm x^{1}+\sum_{j=2}^{d} x^{j}\right| \leq \frac{d-1}{2}+\frac{1}{3}\right\} \\
& K_{2}=\left\{x=\left(x^{1}, x^{2}, \ldots, x^{d}\right):\left|x^{i}\right| \leq \frac{1}{2}\right\}, \quad K_{1} \subset K \subset K_{2},
\end{aligned}
$$

and $\pm\left( \pm \frac{1}{2}, \frac{1}{2}, \ldots, \frac{1}{2}\right) \notin K$, then

$$
N_{\mathrm{L}}(K)=3^{d}-5<3^{d}-3 \leq N_{\mathrm{T}}(K) .
$$

To end this article we raise the following problem:

Problem. Determine

$$
\max _{K \in \mathscr{K}}\left(N_{\mathrm{T}}(K)-N_{\mathrm{L}}(K)\right)
$$

where $\mathscr{K}$ denotes the set of all $d$-dimensional convex bodies.

\section{Acknowledgments}

For the discussion and encouragement, I am obliged to Professor Peter M. Gruber. For the helpful comments, I am obliged to the referees.

\section{References}

1. J. H. Conway and N. J. A. Sloane, Sphere Packings, Lattices and Groups, Springer-Verlag, New York, 1988.

2. H. Groemer, Abschätzungen für die Anzahl der konvexen Körper, die einen konvexen Körper berühren. Monatsh. Math. 65 (1961), 74-81.

3. B. Grünbaum, On a conjecture of H. Hadwiger. Pacific J. Math. 11 (1961), 215-219.

4. G. L. Watson, The number of minimum points of a positive quadratice form, Dissertationes Math. 84 (1971), 1-42.

Received July 14, 1993, and in revised form December 29, 1993. 